\title{
Les stratégies d'accueil et d'accompagnement des nouveaux arrivants déployées par les écoles francophones et les structures communautaires du Nouveau-Brunswick
}

\author{
Lamine Kamano, Aïcha Benimmas et Alexandra Côté
}

Volume 32, numéro 1, 2020

Croisement et intégration des savoirs : deux enjeux dans la recherche francophone en milieu minoritaire

URI : https://id.erudit.org/iderudit/1071931ar

DOI : https://doi.org/10.7202/1071931ar

Aller au sommaire du numéro

Éditeur(s)

Presses universitaires de Saint-Boniface (PUSB)

ISSN

0843-9559 (imprimé)

1916-7792 (numérique)

Découvrir la revue

Citer cet article

Kamano, L., Benimmas, A. \& Côté, A. (2020). Les stratégies d'accueil et d'accompagnement des nouveaux arrivants déployées par les écoles francophones et les structures communautaires du Nouveau-Brunswick. Cahiers franco-canadiens de l'Ouest, 32(1), 35-59.

https://doi.org/10.7202/1071931ar
Résumé de l'article

L'accueil et l'accompagnement des nouveaux arrivants dans leur processus d'établissement constituent l'une des préoccupations des acteurs sociaux et éducatifs de la province du Nouveau-Brunswick. Cet article présente les résultats d'une étude qui vise à comprendre les stratégies d'accueil, de sensibilisation et d'accompagnement déployées par les écoles francophones et les structures d'accueil pour intégrer les familles issues de l'immigration et leurs enfants ainsi que les enjeux entourant le partenariat possible entre ces deux sphères en matière d'intégration des personnes immigrantes. Pour ce faire, une série d'entrevues individuelles ont été menées auprès des directions d'école, du personnel enseignant, des membres d'organismes communautaires et des parents immigrants. L'analyse thématique des données recueillies a permis de dresser un portrait global des différentes stratégies mises en oeuvre par les écoles et les organismes non gouvernementaux (ONG) et communautaires en vue d'accompagner les élèves nouveaux arrivants et leurs familles. Les résultats montrent plusieurs éléments de satisfaction découlant de la collaboration entre les écoles et les organismes communautaires. Toutefois, l'appréciation de ce partenariat tacite par les directions d'écoles et les parents immigrants laisse voir plusieurs lacunes à combler.
Tous droits réservés (c) Centre d'études franco-canadiennes de l'Ouest (CEFCO) et Presses universitaires de Saint-Boniface (PUSB), 2020
Ce document est protégé par la loi sur le droit d'auteur. L’utilisation des services d'Érudit (y compris la reproduction) est assujettie à sa politique d'utilisation que vous pouvez consulter en ligne.

https://apropos.erudit.org/fr/usagers/politique-dutilisation/ 


\section{Les stratégies d'accueil et d'accompagnement des nouveaux arrivants déployées par les écoles francophones et les structures communautaires du Nouveau-Brunswick}

Lamine KAMANO, Université de Moncton Aïcha BENIMMAS, Université de Moncton, Alexandra CÔTÉ, Université de Moncton

\section{RÉSUMÉ}

L'accueil et l'accompagnement des nouveaux arrivants dans leur processus d'établissement constituent l'une des préoccupations des acteurs sociaux et éducatifs de la province du Nouveau-Brunswick. Cet article présente les résultats d'une étude qui vise à comprendre les stratégies d'accueil, de sensibilisation et d'accompagnement déployées par lesécoles francophones et les structures d'accueil pour intégrer les familles issues de l'immigration et leurs enfants ainsi que les enjeux entourant le partenariat possible entre ces deux sphères en matière d'intégration des personnes immigrantes. Pour ce faire, une série d'entrevues individuelles ont été menées auprès des directions d'école, du personnel enseignant, des membres d'organismes communautaires et des parents immigrants. L'analyse thématique des données recueillies a permis de dresser un portrait global des différentes stratégies mises en œuvre par les écoles et les organismes non gouvernementaux (ONG) et communautaires en vue d'accompagner les élèves nouveaux arrivants et leurs familles. Les résultats montrent plusieurs éléments de satisfaction découlant de la collaboration entre les écoles et les organismes communautaires. Toutefois, l'appréciation de ce partenariat tacite par les directions d'écoles et les parents immigrants laisse voir plusieurs lacunes à combler. 
Mots clés: Organismes non gouvernementaux, école francophone minoritaire, partenariat, collaboration, intégration des élèves nouveaux arrivants.

\section{Introduction}

Le contexte francophone du milieu minoritaire canadien en général et celui du Nouveau-Brunswick en particulier est caractérisé par la prise en considération du déclin démographique des communautés francophones fortement menacées par le vieillissement de leurs populations, la faiblesse du taux de natalité, l'exode rural des jeunes et le faible taux des transferts linguistiques (Sall, 2019; Iacovino et Léger, 2013; Landry, 2012). À l'image des autres provinces canadiennes, le Nouveau-Brunswick accueille chaque année des nouveaux arrivants afin de faire face, en partie, à ces différents défis (Kamano et Benimmas, 2017; Traisnel, Violette et Gallant, 2013). A cet effet, plusieurs stratégies de promotion telles que Destination Acadie, ont été mises en place afin d'encourager l'immigration au Nouveau-Brunswick, la cible visée étant de $33 \%$ d'immigrants francophones d'ici 2020 (Sall, 2019). Selon cet auteur, le but de ces initiatives auxquelles plusieurs organismes et institutions des maritimes souscrivent est surtout d'attirer des immigrants francophones. Le gouvernement vise surtout le recrutement de travailleurs qualifiés capables de contribuer à la croissance économique (Paquet, 2016). En plus du Programme des candidats de la province qui découle d'une entente avec le gouvernement fédéral, la province bénéficie depuis 2018 du projet pilote d'immigration au Canada Atlantique, qui permet de combler des besoins en main-d'œuvre des employeurs désignés (Sall, 2018). À cela s'ajoutent la facilitation accordée aux étudiants internationaux quant à l'obtention du statut de résidence permanente après leurs études (Belkhodja, 2012). Au dernier recensement de Statistique Canada, la province du Nouveau-Brunswick a accueilli un total de 865 immigrants d'expression française de 2011 à 2015 toutes catégories confondues, soit 173 immigrants par année en moyenne. La répartition de ces immigrants par catégorie est très inégale, puisqu'on dénombre pour la même période 625 immigrants économiques, 130 réfugiés et personnes protégées et 110 de la catégorie de la famille parrainée (Statistique Canada, 2016). 
Ces statistiques seraient en perpétuelle augmentation depuis ce dernier recensement.

De ce constat, il est indéniable que la province fournit en matière de gouvernance de réels efforts afin d'attirer les immigrants (Sall et Rolland, 2018; Farmer, Bélanger et Cyr, 2013), toutefois leur rétention constitue un autre enjeu fondamental. En vue d'y faire face, il existe présentement plusieurs organismes et associations tels que le Centre d'accueil et d'accompagnement francophone des immigrants du Sud-Est du Nouveau-Brunswick (CAFI), l'Association multiculturelle du Grand-Moncton (MAGMA), la ville de Moncton, the Multicultural Association of Fredericton (MAF), le YMCA et le Centre des nouveaux arrivants de Saint-Jean, the Miramichi Regional Multicultural Association (MRMA) de Miramichi, le Comité d'accueil d'intégration et d'établissement des nouveaux arrivants de la péninsule acadienne (CAIENA) et le Carrefour de l'Immigration Rurale de Saint-Léonard qui offrent des services touchant les domaines d'accueil, d'intégration et d'établissement. Bien que l'ensemble de ces structures communautaires offrent des services à tous les immigrants dans les deux langues officielles de la province, certaines associations telles que le CAFI et celles du milieu rural majoritairement francophone mettent surtout l'accent sur les services aux immigrants francophones. Elles accompagnent ainsi les écoles francophones dans leur processus d'intégration des élèves immigrants et de leurs parents.

$\mathrm{Au}$ Nouveau-Brunswick, comme partout ailleurs, l'intégration des immigrants dans la société d'accueil, plus particulièrement à l'école, s'accompagne de défis liés notamment au placement, à la francisation, à la collaboration avec les familles immigrantes, à la méconnaissance des valeurs et antécédents scolaires des élèves nouveaux arrivants, bref à leur intégration socioscolaire (Kamano et Benimmas, 2017; Kamano, 2014). Toutefois, dans ce processus d'accueil et d'intégration, de nombreux efforts conjoints seraient fournis par des instances et structures concernées ces dernières années. Sur le terrain, ces organismes et l'école francophone collaborent étroitement pour faciliter l'accueil et l'accompagnement des élèves nouveaux arrivants et leurs familles. L'accompagnement et le soutien de cette dernière catégorie $\mathrm{d}$ 'immigrants dans ces milieux soulèvent une gamme complexe de réalités et de facteurs, qui tiennent 
autant à la spécificité des besoins de chaque élève qu'à des caractéristiques propres à la famille et sa trajectoire migratoire. La présente recherche a pour objectifs de : 1) comprendre les stratégies d'accueil et de sensibilisation déployées par les écoles francophones et les organismes d'accueil et d'établissement pour accompagner les familles immigrées et leurs enfants; 2) analyser le partenariat possible qui existe entre les deux sphères en matière d'intégration des élèves immigrants et de leurs familles et 3) porter un jugement sur les résultats d'un tel partenariat.

\section{Cadre de référence}

Il est indéniable que l'intégration sociale des immigrants au sein de la communauté d'accueil joue beaucoup dans leur rétention et que l'école est un espace incontournable dans le processus d'adaptation des enfants immigrants (Kanouté, Duong et Charrette, 2010; Kanouté et Lafortune, 2011). C'est dans cet espace que les jeunes immigrants vivent leur processus d'acculturation et d'apprentissage des nouveaux codes sociaux en vue d'y être acceptés et reconnus en tant qu'individus à part entière (Makarova, 2019). Les chercheurs comme Aydin et Kaya (2017); Qaddour (2017); Lafortune et Kanouté (2011) s'accordent sur le fait que l'expérience de l'immigration est traumatisante pour les enfants immigrants à cause des ruptures avec leur environnement culturel d'origine, des pertes de liens et de repères, des efforts fournis pour l'établissement d'un nouveau réseau social, la compréhension des codes de sociabilité et des changements qui caractérisent la nouvelle vie. Dans ce sens, Lloydetta (2008) explique les causes de la souffrance de ces élèves par trois besoins psychologiques, à savoir : «l'interaction sociale, l'attitude face à l'école et la socialisation. » Ainsi, l'enjeu de l'immigration pour l'éducation réside dans le succès de l'intégration sociale et scolaire de l'élève immigrant. Puisque l'école se trouve liée à sa communauté, elle a besoin de l'aide des acteurs concernés par l'immigration comme les structures d'accueil et d'établissement afin de communiquer avec les familles immigrées et de comprendre leurs conditions de vie au sein de la société d'accueil. Selon Bilodeau et al. (2011, p.37-54) les collaborations école-famille-communauté sont souvent retenues par les politiques et les programmes publics, notamment en éducation, afin de répondre aux défis de 
l'immigration. Ces collaborations se font sous forme de réseaux où différents acteurs organisationnels sontengagés dans des liens collaboratifs. Dans cette logique collaborative, des savoirs et des biens, notamment des fonds obtenus des instances publiques ou privées peuvent être mobilisés par ces structures d'accueil en vue d'accompagner l'école dans le processus d'intégration des élèves immigrants et de leurs familles (Kanouté et Lafortune, 2011). Dans l'action partenariale, ces structures obéissent à la dynamique collaborative qui se réfère aux interactions dans le but d'assurer une meilleure réalisation concertée des interventions (Bilodeau, Bélanger, Gagnon et Lussier, 2009). Or, en contexte francophone minoritaire, cette dynamique collaborative est souvent accompagnée de tensions entre les structures anglodominantes et celles francophones. Le fait de vouloir conserver son espace d'intervention peut entrainer un manque de synergie d'actions entre les différentes structures communautaires en présence.

\section{La dynamique collaborative}

Dans le processus d'intégration des élèves immigrants, l'école entretient des relations étroites avec certains partenaires sociaux et communautaires. Ces relations peuvent revêtir plusieurs aspects selon les objectifs visés, dont les principales sont la collaboration, la concertation, la coopération ou le partenariat (St-Juste, 2017). La collaboration vise le consensus, l'entente dans la mise en œuvre des actions à privilégier, la concertation est basée sur un échange d'idées alors que la coopération s'appréhende dans une perspective d'agir ensemble dans la réalisation d'une tâche (St-Juste, 2017; Périer, 2007). Quant au partenariat, le Comte (2004) le définit comme le résultat

d'une entente réciproque entre des parties qui, de façon volontaire et égalitaire, partagent un objectif commun et le réalisent en utilisant de façon convergente leurs ressources respectives (p.2).

En milieu éducatif, le terme partenariat renvoie à une relation avec différents acteurs extérieurs à l'école qui sont les parents, les collectivités, des structures communautaires, des associations publiques ou privées et même des entreprises en vue d'atteindre un objectif commun en misant sur la convergence des actions à mener (Sarfati, 2013). En tenant compte des acteurs impliqués, il existe plusieurs modèles de 
collaboration bien documentés. En matière de relation écolefamille immigrante, Vatz Laarousi et al., (2008, p. 291-311) ont identifié six modèles, soit l'implication assignée, la collaboration partenariale, la collaboration avec espace de médiation, la collaboration avec distance assumée, la collaboration en quête de visibilité et la collaboration fusionnelle. Ces modèles sont susceptibles de s'opérer selon les acteurs concernés, les objectifs visés, les rôles à jouer et les actions à mettre en œuvre. Dans le cas d'une dynamique collaborative entre l'école et les structures communautaires, les rôles des acteurs impliqués sont complémentaires, évolutifs et parfois changeants selon les situations ou les enjeux. Par exemple, dans son étude sur le suivi scolaire par les familles défavorisées, (Kanouté, 2003) rapporte les propos des parents selon lesquels «il est nécessaire d'accorder une place plus importante aux ressources communautaires pour compléter les efforts déployés par l'école» (p.415).

Les structures d'accueil jouent un rôle important dans l'intégration des familles immigrées et leurs enfants, et ce, grâce aux programmes communautaires qu'elles offrent à ces familles (Rahm, Lachaîne, Martel-René et Kanouté, 2012). Ces chercheurs précisent qu'à travers ces programmes, les familles immigrées et leurs enfants ont accès aux modèles socioculturels du pays d'accueil et reçoivent de l'aide quant aux besoins linguistiques. Dans une autre étude menée auprès des directions d'école, Kanouté et al. (2010) constatent qu'il existe une unanimité quant à la représentation de la nécessité de collaboration entre l'école et les structures communautaires.

D'après Lavoie et Panet-Raymond (2014), cette collaboration porte généralement sur les services offerts aux familles en matière de logement, de recherche d'emploi, de formation continue, de réseautage, de loisirs, de soutien scolaire. La collaboration avec les structures communautaires peut également porter sur l'élargissement du capital social des familles immigrantes (Kanouté et Charrette, André, Rachédi et Rahm, 2014) et la valorisation de la diversité culturelle à travers les activités organisées conjointement avec l'école (Rahm, 2007).

Abondant dans le même sens, les études de Rahm(2012) et Rahm et Gonsalves (2012) mettent en évidence la pertinence des activités sur lesquelles portent les actions des organismes 
communautaires en soutien aux efforts de l'école. Selon ces auteurs, ces activités généralement organisées après les heures de classe sont diverses et orientées sur les intérêts des jeunes immigrants. Au-delà des apprentissages linguistiques et des codes sociaux réalisés, ces activités permettent aux jeunes de «vivre des relations significatives et positives avec des adultes et de côtoyer d'autres jeunes à l'extérieur du cadre scolaire habituel» (p.88). Ce qui peut contribuer au bien-être des jeunes et à leur épanouissement socioscolaire (Rahm, 2010).

Toutefois, les auteurs tels que Kanouté et al. (2014, p. 3150) identifient de nombreux défis liés à cette collaboration dont le respect de l'horaire de l'école, la confidentialité et les règles de fonctionnement.

Au Nouveau-Brunswick, les défis liés à l'intégration des élèvesimmigrants dans lesécoles francophones ontétéégalement identifiés, parmi lesquels la relation écoles-familles immigrées (Benimmas et Kamano, 2009) et la disponibilité des structures d'accueil dans les écoles francophones (Bouchamma, 2009). Dans le but d'aider l'école à relever ces défis, les villes qui accueillent le plus grand nombre d'immigrants telles que Moncton, Fredericton et Saint-Jean se sont dotées de structures d'accueil et d'établissement : le Centre d'Accueil et d'Accompagnement Francophone des Immigrants du Sud-Est du N.-B (CAFI), Multicultural Association of the Greater Moncton Area (MAGMA) à Moncton, Multicultural Association of Fredericton (MAF), le YMCA et le Centre des nouveaux arrivants de SaintJean. Compte tenu de l'importance du phénomène, d'autres localités rurales de la province ont également mis en place des structures d'accueil et d'intégration dont the Miramichi Regional Multicultural Association (MRMA) de Miramichi, le Comité d'accueil d'intégration et d'établissement des nouveaux arrivants de la péninsule acadienne (CAIENA) et le Carrefour de l'Immigration Rurale de Saint-Léonard au nord-ouest de la province. Certains de ces organismes comme le CAFI repensent le concept d'intégration des immigrants en insistant sur les concepts du réseautage, de l'éducation interculturelle et du partenariat.

Étant donné que l'établissement d'un réseau social influe sur l'intégration sociale des immigrants et par ailleurs leur rétention, on peut se questionner sur comment les acteurs 
communautaires (ONG et associations) et scolaires répondent aux besoins d'intégration sociale des nouveaux arrivants et leurs enfants au sein d'une société qui lutte de manière continue pour sa survie culturelle? Comment lesécoles francophones procèdent en termes de stratégies afin de bien accueillir et intégrer les élèves nouveaux arrivants? Puisqu'il est nécessaire de créer un partenariat entre les organismes non gouvernementaux et les écoles en matière d'accueil et d'intégration des élèves nouveaux arrivants étant leur point commun, sur quoi porte un tel partenariat? Quels résultats émergent de ce partenariat?

Ces questions sont d'autant plus cruciales qu'elles touchent non seulement les programmes des organismes non gouvernementaux et les stratégies d'accueil déployées par les écoles francophones du Nouveau-Brunswick en matière d'intégration des nouveaux arrivants, mais toute la dynamique de collaboration entre les trois pôles (parents nouveaux arrivants et leurs enfants, les écoles et les organismes communautaires et non gouvernementaux).

\section{Méthodologie}

La présente recherche adopte le paradigme qualitatif basé sur une méthode d'analyse thématique et interprétative. Les participants sont les intervenants des organismes non gouvernementaux et des associations, des directions d'école, des agents pédagogiques, des enseignants ressource et des parents immigrants. Ils ont été sélectionnés à partir d'un échantillonnage délibéré afin de respecter certains critères utiles aux objectifs de la recherche. L'échantillon comporte un total de 27 participantes et participants répartis comme suit : 6 enseignantes et enseignants ressource, 8 directions scolaires, 6 membres d'ONG et d'associations et 7 parents immigrants. La méthode de cueillette des données se base principalement sur la conduite d'une série d'entrevues semi-dirigées (Savoie-Zajc, 2009).

En effet, une grille d'entrevue a été élaborée pour les acteurs scolaires, une autre pour les membres des ONG et les associations et une dernière grille a été réservée aux parents d'élèves nouveaux arrivants. Ces instruments nous ont permis de collecter des informations pertinentes quant aux stratégies d'accueil et de sensibilisation des parents immigrants et leurs 
enfants, de même que la portée du partenariat entre les structures d'accueil et les écoles sur l'intégration des immigrants et leurs enfants dans les écoles francophones. Le matériel recueilli a été transcrit intégralement et soumis à une analyse de contenu de type thématique et interprétatif respectant la démarche proposée par Paillé et Mucchielli (2012).

\section{Résultats}

Cet article vise à comprendre les stratégies d'accueil et de sensibilisation déployées par les écoles francophones et les organismes d'accueil et d'établissement pour accompagner les familles immigrées et leurs enfants; à analyser le partenariat possible qui existe entre les deux sphères en matière d'intégration des élèves immigrants et de leurs familles et à apprécier les résultats d'un tel partenariat. En effet, les résultats de l'analyse des différentes entrevues réalisées aussi bien avec les acteurs éducatifs qu'avec les membres des organismes communautaires et associations laissent voir un certain nombre de stratégies d'accueil et de sensibilisation généralement utilisées par ces deux instances.

\section{Stratégies d'accueil, de sensibilisation et d'accompagnement}

Les participantes et participants des écoles francophones accueillant le plus grand nombre d'élèves immigrants et ceux des organismes s'accordent sur le fait que plusieurs stratégies sont mises en place dans le but d'accueillir les nouveaux arrivants et de faciliter leur intégration. Bien que ces deux lieux d'intégration se complètent, les stratégies qu'elles déploient semblent différentes et variées.

\section{Stratégies d'accueil, de sensibilisation et d'accompagnement déployées par les écoles}

À l'échelle scolaire, les stratégies mises en place par les directions d'écoles dans le but d'intégrer les élèves nouveaux arrivants varient selon les écoles. Néanmoins, selon les participantes et participants, l'ensemble de ces stratégies portent sur l'accueil des élèves immigrants et de leurs parents au sein de l'école et le placement des élèves nouveaux arrivants.

Ainsi, l'accueil des élèves nouveaux arrivants et leurs parents au sein des écoles se fait de façon différente d'une 
école à l'autre. Il faut noter que parmi les écoles participantes, certaines possèdent des enseignants d'accueil qui jouent un rôle primordial dans l'accueil des élèves nouveaux arrivants et leurs familles, et ce, à travers l'établissement de contacts avec les parents.

Pour les stratégies d'accueil déployées, les directions d'école exigent un certain nombre de documents permettant d'avoir une idée sur les antécédents scolaires, sanitaires et le statut d'immigration de l'enfant. Ce processus délicat et complexe nécessite de la part de la direction d'école un travail méthodique :

Je vais vérifier s'ils ont des documents de scolarité, j'en prends des photocopies et je vais voir l'enseignant à l'accueil et les conseillères en orientation pour avoir une bonne idée de ce que ça veut dire parce que d'un pays à l'autre la scolarité n'est pas toujours faite de la même façon, des fois, nous autres, la notation c'est sur 5, dans d'autres pays c'est sur 20 (DE1)

Il ressort des propos recueillis auprès des directions d'école la planification d'une rencontre avec les parents accompagnés de leur(s) enfant(s) à l'occasion de laquelle des informations importantes sont données aux nouveaux arrivants : «Les parents vont venir nous rencontrer, on s'assoit avec eux et leurs enfants, quand on peut pis on va un peu les informer du fonctionnement de l'école.» (DE2). Au cours de cette rencontre une trousse préparée par le District scolaire contenant des informations pertinentes quant au fonctionnement de l'école francophone est remise à la famille: «On donne toujours une trousse qu'ils peuvent apporter avec eux, c'est une trousse qui explique le transport, les repas, bienvenue à l'école» (DE5). Ainsi, cette première rencontre a comme objectif d'informer les parents du système scolaire afin de les accompagner dans le cheminement scolaire de leur enfant.

En matière de placement, les directions d'écoles affirment avoir mis en place une procédure d'évaluation des prérequis de l'enfant afin de déterminer son niveau scolaire et ses habiletés.

Ce qu'on fait maintenant, on le faisait moins avant, tout de suite, c'est très simple, l'enfant arrive, on essaye de voir avant son niveau si on a besoin, voir ses capacités pis ses habiletés [...] pour pouvoir le placer, d'avoir un placement judicieux (DE4). 
Cependant, bien que cette stratégie semble fonctionner, certaines directions d'écoles ne l'utilisent pas de façon systématique avec tous les élèves nouveaux arrivants.

C'est du cas par cas, c'est tellement différent d'une personne à l'autre donc j'essaye de mieux connaitre cette personne là parce que parmi eux j'ai des élèves qui sont très scolarisés, j'en ai d'autres qui le sont très peu, ça fait que je ne peux pas dire que c'est un one size fits all comme ils disent là (DE1).

Cette évaluation préliminaire concerne notamment certaines matières fondamentales telles que le français, les mathématiques voire la physique pour les élèves du secondaire. La vérification se fait essentiellement à l'écrit, mais parfois à l'oral dans certains cas. L'école est amenée dans certains cas où l'élève possède un haut niveau de scolarité, à procéder à une vérification de façon orale afin d'assurer un meilleur placement :

On a demandé à l'élève d'évaluer ce qu'il avait touché à l'intérieur d'un ou des programmes d'études parce que c'était quelqu'un qu'on savait qu'il fallait qu'on lui donne de cours de niveaux élevés, mais quels cours? On ne savait pas exactement c'était où la meilleure place (DE1).

Pour certains répondantes et répondants, cette entrevue informelle vise à recueillir certaines informations utiles sur les antécédents scolaires voire même le cheminement de l'élève. Pour ce faire, une des écoles va jusqu'à s'informer auprès des autorités éducatives du pays d'origine de l'élève. Dans ce processus de détermination du niveau de placement des élèves nouveaux arrivants, les répondantes et répondants sollicitent également la contribution des parents afin de comprendre en profondeur le parcours spécifique de l'enfant. La collaboration avec la famille est incontournable pour bien déterminer le niveau de scolarité de l'élève :

Je vais souvent parler avec la famille parce que même si on fait des évaluations en français ou en mathématiques, ça ne donne pas toujours le vrai portrait (DMSCT).

À l'issue de ce processus complexe d'inscription et d'évaluation des acquis de l'enfant, les écoles mettent également en œuvre une série de stratégies visant à sensibiliser et à accompagner les nouveaux arrivants dans leur processus d'intégration. 
Toutefois, il n'existe pratiquement pas de programmes structurés de sensibilisation et d'accompagnement au sein des écoles. Chaque école utilise ses propres stratégies de sensibilisation selon les initiatives de la direction. À cet effet, certaines écoles initient des sessions de formation ponctuelles de sensibilisation destinées au personnel enseignant généralement animées par l'enseignant à l'accueil alors que d'autres organisent des soirées multiculturelles et des kermesses. De plus, certaines directions interrogées procèdent à l'affichage des drapeaux des pays d'origine des élèves immigrants présents à l'école comme une façon de sensibiliser à la diversité qui y règne. Certaines écoles intègrent la sensibilisation à la diversité ethnoculturelle dans certaines matières, telles que le cours de formation personnelle et sociale (FPS) et celui des sciences humaines :

Dans le programme d'études, surtout en formation personnelle et sociale et en sciences humaines, les programmes sont plus ou moins basés sur la diversité culturelle. Alors comme direction je présume que c'est travaillé, ou que c'est abordé ou que le matériel qui est présenté est un matériel ouvert ... qui va montrer justement la diversité autant culturelle que ... personnel (DE4).

Au sein de la même école et dans bien d'autres, les journées spéciales de sensibilisation sont également initiées par la direction. Dénommée la journée Bleue, elle est organisée chaque dernier vendredi du mois avec pour objectif la sensibilisation aux différences, à l'intimidation à travers des thèmes abordés.

Quant à l'accompagnement des élèves nouveaux arrivants, il s'effectue à deux niveaux : scolaire et social. Dans le premier cas, c'est surtout ceux qui ont des difficultés dans la langue d'enseignement et dans certaines matières spécifiques comme les mathématiques qui sont concernés. Le district scolaire affecte à cet effet des ressources humaines telles que des moniteurs de langue dans certaines écoles afin d'accompagner le personnel enseignant. Par ailleurs, la stratégie de jumelage avec un élève ou une personne-ressource est une pratique courante afin que l'enfant puisse être assisté dans son processus d'intégration scolaire. Il y a également des directions d'école qui développent un plan d'intégration scolaire à long terme susceptible d'accompagner les nouveaux arrivants dans leur cheminement d'apprenants : 
Ce qui peut être particulier, c'est que souvent au niveau académique, il y a de grands écarts. Ce qui fait que c'est souvent utile de développer un plan qui va ou qui peut les aider à fonctionner dans le système ici, mais en sachant que ça va prendre un an, deux ans avant qu'il puisse s'adapter (DE4).

Dans les écoles qui reçoivent le plus grand nombre d'élèves immigrants, les enseignantes et enseignants à l'accueil encadrent les élèves nouveaux arrivants sur le plan scolaire, mais surtout sur le plan de l'apprentissage des valeurs culturelles canadiennes en général et acadiennes en particulier.

Les intégrer sur le plan scolaire ce n'est pas évident parce que le fonctionnement de l'école ici n'est pas forcément le même que ce qu'ils ont vécu chez eux, donc dans l'intégration sociale quand ils sont ici, ils restent avec moi pendant un semestre, j'essaye de suivre comment ça fonctionne dans leurs classes, est ce qu'ils ont des amis et est-ce qu'ils, aussi j'essaye quand même de les faire connaitre à les valeurs d'ici parce que souvent ils sont timides et les enfants ici ne font pas aussi le premier pas vers eux (EE3).

Sur le plan social, certaines directions d'école procèdent par des stratégies qui consistent à impliquer les nouveaux arrivants dans divers comités scolaires (ex.: comité de danse) alors que d'autres organisent des activités sportives parascolaires. Elles sont le plus souvent appuyées par les structures communautaires qui de leur côté déploient plusieurs stratégies selon leur champ d'intervention.

\section{Stratégies d'accueil, de sensibilisation et d'accompagnement déployées par les structures non gouvernementales}

$\mathrm{Au}$ Nouveau-Brunswick, bien que l'immigration soit un phénomène récent, on a recensé plusieurs organismes communautaires et associations œuvrant dans l'accueil et l'intégration des immigrants dont, entre autres, le MAGMA, le CAFI, le CIR (St. Léonard) et le YMCA (St. Jean). Ces organismes à but non lucratif orientent, selon les participantes et participants, leurs activités, notamment dans le domaine de l'accueil, de la sensibilisation et de l'accompagnement à l'adaptation des nouveaux arrivants. En effet, en matière d'accueil par exemple, ces structures développent plusieurs stratégies qui concernent aussi bien les services à l'arrivée qu'à l'installation. 
Quand nous recevons une famille, on a une panoplie de services pour ces familles. À l'arrivée à l'aéroport (jusqu'à 2 ans) on signe l'entente pour les supporter émotionnellement donc, on va les accueillir à l'aéroport avec les moyens du carrefour (de nos bailleurs de fonds), on cherche des maisons. On les aide à faire l'épicerie, on cherche un médecin pour eux, on cherche les écoles pour leurs enfants (AA3).

Les stratégies d'accueil mises en œuvre par les organismes communautaires peuvent être regroupées en deux catégories: les activités d'accueil et d'aide au placement des élèves nouveaux arrivants. Pour accueillir les nouveaux arrivants, ces organismes mettent en place des activités de bienvenue auxquelles participent les membres de la société d'accueil comme bénévoles recrutés par les coordinateurs de connexion communautaire.

On a deux personnes qui travaillent à ce rôle pour aller chercher les nouveaux arrivants à l'aéroport, leur trouver un appartement, les aider avec les épiceries de base, la valeur de monnaie canadienne, ouvrir un compte de banque, les cartes d'assurance médicale, les numéros d'assurance sociale, des applications pour les cartes de résidence permanente, des références dans divers services que la communauté offre ainsi que divers services au sein de notre association (AA2).

Parallèlement à ces différentes activités d'accueil, les structures communautaires assistent aussi les parents d'élèves nouveaux arrivants dans le processus de choix d'école, d'inscription et de placement de l'enfant. À l'image des écoles, les organismes et associations mettent en œuvre plusieurs stratégies de sensibilisation et d'accompagnement des nouveaux arrivants. À la différence des écoles, les services offerts semblent plus structurés. En plus des activités socioculturelles qu'elles organisent, ces structures communautaires font le plus souvent des présentations sur la diversité ethnoculturelle. Ces présentations s'adressent aux écoles, aux professionnels qui travaillent avec les jeunes et aux étudiants en éducation à la petite enfance afin de les sensibiliser aux différences culturelles tout en leur faisant comprendre les différents défis auxquels le nouvel arrivant fait face dans son processus d'intégration à la société d'accueil. 
En vue d'accompagner les nouveaux arrivants dans leur processus d'intégration, les organismes communautaires utilisent plusieurs stratégies regroupant des services d'aide à la recherche d'emploi, des cours de langues (français et anglais), des services d'établissement (jumelage, activités sociales, programme d'habiletés de vie sécuritaire, alimentaire et hygiénique ainsi que divers ateliers d'intégration). Dans bien des cas, la mise en œuvre de ces actions donne lieu à des partenariats entre l'école et les organismes communautaires.

\section{Partenariat entre les écoles et les structures d'accueil et d'établissement}

L'analyse des propos recueillis auprès des différentes directions d'école révèle qu'au Nouveau-Brunswick le partenariat entre les écoles et les structures communautaires n'est pas inscrit dans les documents définissant la mission et la politique de fonctionnement des écoles. Tous les partenariats tacites actuellement en vigueur relèvent des initiatives des directions d'école ou des districts scolaires.

\section{Dynamique du partenariat}

Bien qu'iln'existe pas de partenariats formels, les directions d'écoles et les districts scolaires francophones affirment qu'ils collaborentaveclesorganismescommunautairesdéjàmentionnés et certaines organisations non gouvernementales (ex. Clubs garçons et filles, Maison des jeunes, Jeunes d'abord, Capitol, etc.) ainsi que des institutions d'enseignement postsecondaire (Université de Moncton, Collège communautaire). Ce partenariat porte sur plusieurs aspects, notamment le partage d'informations, l'inscription et le placement scolaire des élèves nouveaux arrivants, l'allocation d'interprètes et de bénévoles, l'aide aux devoirs pour certains élèves, les services de garde d'enfants à des occasions spécifiques. Certaines écoles affirment avoir une collaboration particulière avec le MAGMA lorsqu'il s'agit des questions de violences familiales.

Par ailleurs, outre la collaboration avec les organismes communautaires, les districts et les écoles francophones entretiennent également des relations de collaboration avec certaines organisations non gouvernementales telles que la Croix-Rouge, les Clubs garçons et filles, la Maison des jeunes, les Jeunes d'abord, le Capitol, la bibliothèque de Dieppe, les 
Chevaliers de Colomb et le Katimavik. Selon les directions d'école, ces organismes accompagnent les districts scolaires et les écoles dans le processus d'intégration scolaire et sociale des élèves nouveaux arrivants à travers les dons de documents divers, de vêtements, d'aliments, mais aussi l'assistance quant à l'organisation de certaines activités récréatives et sportives.

\section{Appréciation du partenariat entre les écoles et les structures communautaires}

Le résultat du partenariat entre les écoles et les structures communautaires a été envisagé en tenant compte du point de vue des directions d'école et de celui des parents d'élèves immigrants. À prime abord, les directions d'écoles concernées par cette étude se disent satisfaites des prestations des structures d'accueil en vue d'une intégration harmonieuse des élèves nouveaux arrivants. «On peut ne pas dire qu'on n'est pas satisfait. Je pense que ce qu'elles font, elles le font bien pour nous aider.» (DE2).

Cependant, selon les directions interrogées, la collaboration entre les écoles francophones et les organismes communautaires comportent aussi bien des éléments de satisfaction que des points à améliorer en vue d'optimiser les résultats. Plusieurs éléments de satisfaction ont été ressortis par les directions d'école, tels l'aide à l'accueil, à l'encadrement et l'accompagnement des nouveaux arrivants. D'après ces participants, le travail des associations complète les efforts de l'école notamment en ce concerne les contacts écoles-parents immigrants.

Pour moi, quand on réussit à faire ça, à une famille, de l'accompagner comme ça, à partir de la maison, à l'école pis là tu fais ça quelques fois les premières rencontres parent-maitre, je vais aller avec toi, te montrer comment ça se passe, tu as besoin c'est impossible de demander ça à une personne dans toutes les écoles (DE4).

Par ailleurs, même si les directions d'école semblent satisfaites des différentes prestations des structures communautaires, il n'en demeure moins qu'elles sollicitent d'avantage leur implication plus spécifiquement en ce qui a traità 
la gestion des conflits école-famille immigrante, à l'optimisation de la communication entre ces deux acteurs à travers une assistance à l'élaboration des trousses d'informations préalables destinées aux familles et aux élèves nouveaux arrivants. En vue d'assurer l'efficacité de cette collaboration, les directions d'école interrogées suggèrent une meilleure communication entre les écoles et les organismes communautaires afin de mieux connaitre les ressources disponibles et par conséquence, cibler les tâches et les niveaux d'intervention :

C'est une communication qui devrait être plus fréquente. Peut-être on devrait aller voir ben c'est quoi leurs services, qu'est-ce qu'ils offrent, c'est quoi leurs attentes. Et si nous on a besoin du soutien scolaire, qu'est-ce qu'eux peuvent offrir par rapport à ça, de comprendre où nous on s'insère dans le processus pis, c'est quoi leur rôle, eux autres, par rapport au niveau scolaire, de mieux comprendre quels sont les rôles à chacun pour peut-être mieux les accomplir. (DE2)

Du côté des parents d'élèves nouveaux arrivants, ce partenariat tacite entre les écoles et les organismes s'avère très bénéfique d'autant plus qu'il leur permet non seulement d'entrer facilement en contact avec l'école de leur enfant, mais aussi de s'intégrer à la société d'accueil à travers les activités et les services dont ils bénéficient. Les parents immigrants se disent satisfaits de ce partenariat qui les rassure de plus en plus dans leur processus d'intégration à la société d'accueil. Le sentiment de sécurité que le partenariat semble susciter chez ces parents immigrants, peut indiquer une certaine fragilité expliquée par un manque d'éducation ou une situation économique défavorisée.

Moi personnellement ça m'encourage beaucoup. Je vois que je ne suis pas seul, je vois que si je tombe il y a quelqu'un qui va me relever. Donc, c'est-à-dire que l'école est là, le MAGMA est là, avec les deux, je me sens en sécurité (P3).

Cette collaboration permet à d'autres parents de connaitre leurs devoirs et leurs droits: "parce qu'ils t'aident beaucoup à connaitre qu'est-ce que tu dois faire, qu'est-ce que tu ne dois pas faire» (P1). Puisqu'ils considèrent les organismes communautaires et l'école comme deux réseaux sociaux qui offrent l'accès à la société d'accueil, les parents immigrants interrogés souhaitent être mieux outillés quant aux démarches 
de recherche d'emploi à leur arrivée. Ainsi, ils sollicitent plus d'activités d'intégration en français et d'accompagnement dans le processus d'intégration de leurs enfants.

\section{Discussion}

À la différence des provinces comportant de grands centres urbains généralement choisis par les immigrants, la province du Nouveau-Brunswick peine encore à multiplier les organismes dévoués à la cause des immigrants d'expression française. Les structures existantes sont de création récente suite aux besoins pressants que pose l'arrivée des immigrants. Il n'existe pratiquement pas de programmes ministériels structurés visant à répondre adéquatement aux besoins d'accueil et d'intégration des élèves nouveaux arrivants. Toutes les stratégies mises sur pied par ces deux acteurs socioéducatifs (écoles et organismes communautaires) relèveraient des initiatives isolées des districts scolaires, des écoles ou d'organismes non gouvernementaux.

De plus, le partenariat entre les écoles et les structures communautaires n'étant pas inscrit dans les documents définissant la mission et la politique de fonctionnement des écoles, tous les partenariats présentement mis en œuvre relèvent également d'initiatives des directions d'école ou des districts scolaires. Ces partenariats portent souvent sur le partage d'information, l'inscription et le placement scolaire des élèves nouveaux arrivants, l'allocation d'interprètes et de bénévoles, l'aide aux devoirs pour certains élèves, les services de garde d'enfants à des occasions spécifiques et la francisation.

Les organismes d'accueil servent parfois de pont entre les écoles et les familles immigrantes dans le processus de choix de l'école, d'inscription et d'intégration à la vie scolaire des enfants. À la différence des ONG présents dans d'autres provinces comme l'Ontario, le Québec et l'Alberta, il manque une certaine synergie d'actions entre les structures communautaires et les ONG œuvrant dans l'accueil et l'intégration des immigrants et de leurs enfants. Ce manque de synergie peut s'expliquer par la compétitivité due à la réalité francophone minoritaire. Les structures communautaires et ONG étant majoritairement anglophones ou bilingues, les quelques rares structures à vocation francophone auraient tendance à s'occuper seulement des écoles francophones, ce qui se traduit par des tensions. 
Ce résultat corrobore les conclusions des études de Kanouté et al. (2011) et de Millet et Thin (2007) selon lesquelles la dynamique partenariale école-structures communautaires qui fait appel à des échanges d'expertise est un lieu de tensions qui peuvent être géré par une simple volonté de "partage et de reconnaissance réciproque» (p.418). Toutefois, les efforts de l'école seraient davantage manifestes si elle reconnaissait les ressources qu' offrent les organismes communautaires dans une perspective de complémentarité (Kanouté et al., 2011, p. 407421). Car, la «prise en charge des besoins complexes des familles immigrantes impose une dynamique de collaboration, voire de partenariat, entre l'école et des ressources comme les organismes communautaires» (Kanouté et al., 2011 p. 418). Mieux, comme le mentionnent Farmer et al. (2013, p.1-20) et Jacquet et Masinda, (2014), l'accueil et l'accompagnement des personnes immigrantes nécessitent l'élaboration d'une approche globale, flexible et concertée englobant à la fois les autorités éducatives, les écoles et les structures communautaires.

\section{Conclusion}

Cette recherche visait à cerner les stratégies d'accueil et de sensibilisation déployées par les écoles francophones $\mathrm{du}$ Nouveau-Brunswick et les structures communautaires pour intégrer les familles immigrées et leurs enfants ainsi que les résultats du partenariat possible entre ces deux sphères en matière d'intégration des élèves immigrants et de leurs familles.

L'analyse thématique a permis d'identifier plusieurs stratégies informelles mises en place par les écoles, les associations et les ONG. Ces stratégies portent généralement sur l'accueil (rencontres avec les parents, trousses d'informations, service d'accueil); le placement (évaluation du niveau scolaire, renseignements sur les antécédents socio-scolaires); la sensibilisation (conférence de sensibilisation, de la diversité, rencontre de sensibilisation du personnel) et l'accompagnement des nouveaux arrivants (activités parascolaires, francisation et jumelage). De leur côté, les structures communautaires utilisent plusieurs stratégies regroupant des services d'aide à la recherche d'emploi, des cours de langues (français et anglais), des services d'établissement (jumelage, activités sociales, programme d'habiletés de vie sécuritaire, alimentaire et hygiénique) ainsi 
que divers ateliers d'intégration susceptibles de favoriser l'accueil et l'accompagnement des nouveaux arrivants.

Quant à l'appréciation de ce partenariat par les directions et d'école et les parents immigrants, les résultats indiquent plusieurs éléments de satisfaction découlant de la collaboration entre les écoles et les organismes communautaires, même s'il reste des éléments à améliorer tels que l'aide à l'accueil et à la conception de trousses, l'implication dans la gestion des conflits école/famille, la meilleure communication écoleONG, l'accompagnement dans la recherche d'emplois et la multiplication d'activités en français tout en mettant l'accent sur l'intégration des enfants. Cependant, il convient de noter que cette satisfaction reliée à ce partenariat peut ne pas être la même pour les familles immigrées ayant un haut niveau d'éducation et une autonomie socioéconomique.

En résumé, pour la majorité des nouveaux arrivants de la province les structures d'accueil et d'accompagnement sont le premier moyen de contact avec la société d'accueil, qu'il s'agisse de la recherche de logements, de l'apprentissage des langues officielles, des services de santé, de la recherche d'emploi ou de la scolarisation des enfants. C'est aussi à ces organismes qu'ils ont le plus souvent recours afin de bâtir leur réseau social avec les associations religieuses et ethnoculturelles.

Toutefois, selon les résultats, il manque une certaine synergie d'actions entre les différents organismes et associations communautaires qui s'occupent de l'accueil et de l'accompagnement des nouveaux arrivants au NouveauBrunswick. Cela s'explique par les tensions inhérentes au milieu francophone minoritaire où les structures communautaires sont majoritairement anglophones ou bilingues. Les rares organismes à vocation francophone ont tendance à s'occuper seulement des écoles francophones au sein desquelles il n'existe pratiquement pas de programmes officiellement structurés de sensibilisation et d'accompagnement des nouveaux arrivants. Chaque école utilise ses stratégies de sensibilisation selon ses propres initiatives. 


\section{RÉFÉRENCES}

AYDIN, Hasan, GUNDOGDU, Mahmut et AKGUL, Arif (2019) «Integration of Syrian Refugees in Turkey: Understanding the Educators' Perception», Journal of International Migration and Integration» Revue de l'intégration et de la migration international, January 2019.

AYDIN, Hasan et KAYA, Yeliz (2017) «The educational needs of and barriers faced by Syrian refugee students in Turkey: a qualitative case study», Intercultural Education, vol. 28, $\mathrm{n}^{\circ} 5$, p. $456-473$.

BELKHODJA, Chedly, TRAISNEL, Christophe et WADE, Mathieu (2012) «Typologie des communautés francophones en situation minoritaire du Canada», Rapport de recherche. Ottawa, Citoyenneté et Immigration Canada.

BELKHODJA, Chedly (2010) «Vers une communauté plus accueillante? Quelques observations dans la région de Moncton» En ligne : http://canada.metropolis.net/pdfs/welcoming_moncton_f.pdf

(2012). «La dynamique migratoire des étudiants internationaux et les politiques d'immigration dans cinq fédérations», dans Chedly BELKHODJA et VATZ LAAROUSSI, Michèle (dir.) Immigration hors des grands centres. Enjeux, politiques et pratiques dans cinq États fédéraux: Australie, Belgique, Canada, Espagne, Suisse, Paris, Harmattan, p. 173-196.

BENIMMAS, Aïcha et KAMANO, Lamine (2009) «Comment les futurs enseignants et enseignantes perçoivent-ils l'intégration de l'élève immigrant à l'école francophone en milieu minoritaire au Nouveau-Brunswick» L'établissement international au Canada, vol. 23, no 2, p. 6-9.

BILODEAU, Angèle, BÉLANGER, Robert, GAGNON, Francis et LUSSIER, Nathalie (2009) «School-community collaborations and measures supporting academic achievement in two underprivileged Montreal neighbourhoods: An evaluation of processes and effects ", dans DESLANDES, R. (dir.), International Perspectives on Contexts, Communities and Evaluated Innovative Practices: Family-School-community Partnerships, London, Taylor \& Francis Books, p. 143-161.

BILODEAU, Angèle, LEFEBVRE, Chantal, DESHAIES, Suzanne, GAGNON, Francis, BÉLANGER, Robert, COUTURIER, Jean Yves, POTVIN, Maryse et CARIGNAN, Nicole (2011) «Interventions issues de la collaboration école-communauté dans quatre territoires montréalais pluriethniques et défavorisés.» Service social, vol. 57, nº 2, p.37-54.

https://doi.org/10.7202/1006292ar. 
BOUCHAMMA, Yamina (2009) «Francisation, scolarisation et socialisation des élèves immigrants en milieu minoritaire francophone du Nouveau-Brunswick: quels défis et quelles perspectives?» Document repéré à http://community.smu.ca/atlantic/documents/.

COMTE, Daniel (2004) «La notion de partenariat» Les cahiers pédagogiques, $\mathrm{n}^{\circ}$ 421. Récupéré de http://eedd.ac-montpellier. $\mathrm{fr} / \mathrm{IMG} / \mathrm{pdf} /$.

FARMER, Diane, BÉLANGER, Nathalie et CYR, Lori-Ann (2013) «Pour une gouvernance renouvelée dans le secteur de l'immigration au Nouveau-Brunswick : représentations et mobilisation autour d'un instrument d'action publique.» Revue Gouvernance, vol. 10, nº 1. https://doi.org/10.7202/1038897ar.

GOUVERNEMENT DU NOUVEAU-BRUNSWICK (2014) Plan d'action pour favoriser l'immigration francophone au Nouveau-Brunswick. Mettre nos ressources à l'œuvre, Fredericton, Stratégie de croissance démographique.

IACOVINO, Raffaele et LÉGER, Rémi (2013) «Francophone minority communities and immigrant integration in Canada: rethinking the normative foundations», Canadian Ethnic Studies/ Études ethniques canadiennes, vol. 45, $n^{\text {os }} 1-2$, p. 95-114.

JACQUET, Marianne et MASINDA, Mambo Tadu (2014) «Réflexions sur la notion d'intégration scolaire des jeunes immigrants», Revue internationale d'études canadiennes, vol. 50, p. 277-295, DOI http://dx.doi.org/10.3138/ijcs.2014.015.

LAVOIE, Jocelyne et PANET-RAYMOND, Jean (2014) La pratique de l'action communautaire, $3^{\text {e }}$ édition actualisée. PUQ.

KAMANO, Lamine (2014) «La diversité ethnoculturelle au sein de l'école francophone du Nouveau-Brunswick: croyances et pratiques pédagogiques», Thèse de doctorat (inédit), Université de Moncton.

KAMANO, Lamine et BENIMMAS, Aïcha (2017) «La diversité ethnoculturelle et les enjeux de la pratique pédagogique à l'école francophone minoritaire du Nouveau-Brunswick» Revue Minorités linguistiques et sociétés, Université de Moncton, Canada.

KANOUTÉ, F. (2003) Les parents de milieux défavorisés et l'accompagnement scolaire de leurs enfants, Montréal, QC : Comité de gestion de la taxe scolaire de l'île de Montréal.

KANOUTÉ, Fasal, CHARRETTE, Josée, VIERGINAT, André, RACHÉDI, Lilyane et RAHM, Jrène (2014) «Les dynamiques d'établissement, les enjeux scolaires et les ressources 
communautaires. Le point de vue de parents d'origine haïtienne.» Diversité urbaine, vol. 14, n 2, p. 31-50.

KANOUTÉ, Fasal, LAFORTUNE, Gina (2011) «La réussite scolaire des élèves d'origine immigrée : réflexions sur quelques enjeux à Montréal.» Education et francophonie, vol. 39, nº 1, p. 80-92.

KANOUTÉ, Fasal, RACHÉDI, Lilyane, RAHM, Jrène., TOUSSAINT, Pierre, XENOCOSTAS, Spyridoula et LAFORTUNE, Gina (2010, mai) «Intégration des savoirs disciplinaires en formation universitaire pour tenir compte de l'articulation des logiques d'intervention dans et autour des écoles», Communication présentée au $26^{\mathrm{e}}$ congrès de l'Association Internationale de Pédagogie Universitaire (AIPU), Rabat, Maroc.

KANOUTÉ, Fasal, VIERGINAT, André, CHARRETTE, Joslyne, LAFORTUNE, Gina, LAVOIE, Annick et GOSSELINGAGNÉE, Justine (2011) «Les relations école-organisme communautaire en contexte pluriethnicité et de la défavorisation.» Mcgill journal of education, vol. 46, $\mathrm{n}^{\circ} 3$, p. 407-421.

KANOUTÉ, Fasal., DUONG, Leatitia et CHARETTE, Josée (2010) «Quotidien, réussite et projet scolaires des enfants : le point de vue de trois familles immigrantes» dans PRONOVOST, G. (dir.), Familles et réussite éducative, Québec, QC : Presses de l’Université du Québec, p. 41-54.

MAKAROVA, Elena (2019) «Acculturation and school adjustment of minority students: school and family-related factors» Intercultural Education, vol. 30, n 5, p. 445-447.

MILLET, Mathias et THIN, Daniel (2007) «L'école au cœur de la question sociale entre altération des solidarités sociales et nouvelles affectations institutionnelles» dans PAUGAM, S. (dir.), Repenser la solidarité. L'apport des sciences sociales, Paris, PUF, p. 687-703.

PAILLÉ, Pierre et MUCCHIELLI, Alex (2012) L'analyse qualitative en sciences humaines et sociales, 3 ed., Paris, A.Colin

PAQUET, Mireille (2016) La fédéralisation de l'immigration au Canada, Montréal, Les Presses de l'Université de Montréal.

QUAICOE, Lloydetta (2008) «Negotiating a sense of belonging: immigrant and refugee children in a new school environment in Canada» International Journal of Diversity in Organisations, Communities and Nations, vol. 5, n 6, p. 197-204.

QADDOUR, Kanana (2017) «Educating Syrian refugees in turkey» Récupéré de http://carnegieendowment.org/sada/74782. 
RAHM, Jrène et GONSALVES, Allison (2012) «To understand the News you need Science! Stories of Girls' learning Trajectories and positioning Work within and beyond an After school Science Program», dans VARELAS, M. (dir.), Identity Construction and Science Education Research : Learning, teaching.

RAHM, Jrène LACHAÎNE, Audrey, MARTEL-RENY, MariePaule et KANOUTÉ, Fasal (2012) «Le rôle des organismes communautaires dans la réussite scolaire et le développement identitaire des jeunes issus de l'immigration, Diversité urbaine, vol. 12, no 1 , p. 87-104.

RAHM, Jrène (2007) «Learning and Becoming across Time and Space : A Look at Learning Trajectories Within and Across Two Inner-City Youth Community Science Programs », dans Roth, W.-M. et TOBIN, K. (dir.), Science, Learning, and Identity: Sociocultural and Cultural-Historical Perspectives, Rotterdam, Sense Publisher, p. 63-79.

(2010) Science in the Making at the Margin : A Multisited Ethnography of learning and becoming in an Afterschool Program, a Garden, and an Upward Bound Program, Rotterdam, Netherlands, Sense Publisher.

(2012) «Collaborative Imaginaries and Multi-Sited Ethnography : Space-Time Dimensions of Engagement in an Afterschool Science Program for Girls», Ethnography and Education, vol. 7, $\mathrm{n}^{\mathrm{o}} 2$, p. 247-264.

SALL, Leyla et BOLLAND, Benoit (2018) «L'immigration française et belge à Moncton, une remise en cause de la prévalence de la "loi" économique des dynamiques migratoires», Revue d'études sur le Nouveau-Brunswick/Journal of New Brunswick Studies, vol. 10, p. 59-81.

SALL, Leyla (2018) «Niches d'emplois et barrières d'accès au marché du travail des nouveaux immigrants francophones en Acadie des Maritimes.» Rapport de recherche, Moncton, Immigration, Réfugiés Citoyenneté Canada.

(2019) «Les politiques publiques d'immigration francophone en Acadie du Nouveau-Brunswick : entre incomplétude institutionnelle et succès symbolique» Lien social et Politiques, no 83, p. 272-294, https://doi.org/10.7202/1066094ar.

SARFARI, Jean-Jacques (2013) «Réflexions générales sur la politique de partenariat à l'école» Éducation et socialisation, $\mathrm{n}^{\circ} 34$.

SAVOIE-ZAJC, Loraine (2009) «L'entrevue semi-dirigée» dans GAUTHIER, B. (dir.) Recherche sociologique. De la problématique à la collecte des données, Québec: Presses de l’Université du Québec, p. 337-360. 
STATISTIQUE CANADA (2016) Immigration et diversité ethnoculturelle, Gouvernement du Canada.

ST-JUSTE, Pascale, (2017) «Le plan d'intervention, dispositif de communication entre l'école et la famille immigrante et enjeu de leur partenariat», Mémoire en travail social inédit, UQAM.

TRAISNEL, Christophe, VIOLETTE, Isabelle et GALLANT, Nicole (2013) «Les minorités francophones canadiennes face à l'immigration: particularismes et représentations de la diversité ethnoculturelle dans les milieux associatifs», Minorités linguistiques et société, vol. 1, n², p. 9-28.

TRAISNEL, Christophe (2012) «Protéger et pacifier. La politique officielle de bilinguisme canadien face aux risques de transferts linguistiques et de contestation communautaire», International Journal of Canadian Studies, vol. 45-46, p. 69-89.

VATZ LAAROUSSI, Michelle, KANOUTÉ, Fasal, et RACHÉDI, Lilyane (2008) «Les divers modèles de collaborations familles immigrantes-écoles : de l'implication assignée au partenariat», Revue des sciences de l'éducation, vol. 34, n 2, p. 291-311. 